\title{
NONTOPOLOGICAL SOLITONS
}

\author{
R, Friedberg
}

Bomard College and Columbia University, New York, N. Y. 10027

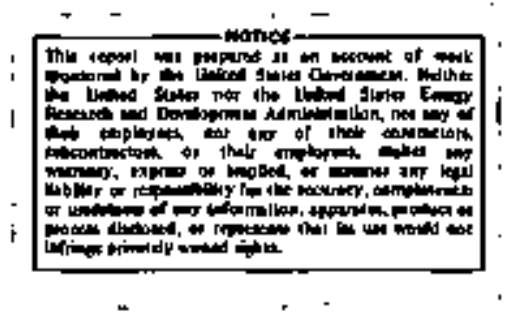

A ralk presented at the Obis Scientiae, Coral Gables, January 1977.

This research was supported in port by the U.S. Energy Reseorch and Development Administrotion. 


\section{DISCLAIMER}

This report was prepared as an account of work sponsored by an agency of the United States Government. Neither the United States Government nor any agency Thereot, nor any of their employees, makes any warranty, express or implied, or assumes any legal liability or responsibility for the accuracy, completeness, or usefulness of any information, apparatus, product, or process disclosed, or represents that its use would not infringe privately owned rights. Reference herein to any specific commercial product, process, or service by trade name, trademark, manufacturer, or otherwise does not necessarily constitute or imply its endorsement, recommendation, or favoring by the United States Government or any agency thereof. The views and opinions of authors expressed herein do not necessarlly state or reflect those of the United States Government or any agency thereof. 


\section{DISCLAIMER}

Portions of this document may be illegible in electronic image products. Images are produced from the best available original document. 
I shall be describing mainly the work carried out over the last few years by T. D. Lee and a number of colloborators, including G. C. Wick, N. H. Christ, M. Margulies, A. Sirlin, and myself.

This work is guided by the premise that we already know enough bosic physics to explain the extended structure of hodrons in terms of a conventional local field theory. On the evidence of $(e, N)$ and $(v, N)$ reactions, we expect local field theory to hold good down to $\sim 10^{-16} \mathrm{~cm}$. Therefore the hodrons extending over $10^{-13}$ should be bound states of a more elementary object. In view of the successes of group theory, we expect the elementory objects to be colored quarks, on the basis of deep inelastic e-scaltering, the quarks should have no intemal structure larger than $10^{-16} \mathrm{~cm}$ and can therefore be regarded as the elementory excitations of a Fermi field.

If everything is this eosy, what stands in the way? Since the quarks hove not been seen in isolation, they must be supposed either extremely massive or unstable; in the latter case they are at leost as massive as the proton. Thus o three-quark nuclean or two-quark meson must be strongly bound, with relativistic effects prominent. It is the difficulty of handling bound states in relotivistic quantum field theory that hos hampered development of a simple conventional model of hodrons.

The study of solitons offers a new deporture for this problem, by suggesting a different $0^{\text {th }}$-order approximation from the one most often studied in the past. The principle is illustroted by the case of a quantum mechanical particle moving in two dimensions under the centrolly symmetric and quasi-hamonic potential $V=\frac{1}{2} r^{2}\left[(I-g r)^{2}+\Delta^{2} g^{2}\right]$. In Fig. I we graph the effective radial potential 
$V_{\ell}=V+\ell^{2} / 2 r^{2}$ against $r^{2}$ for severol values of $\ell$. In general there moy be two minimo, one at small $r^{2}$ and one at lorge $r^{2}$.

When $Q$ exceeds a critical value $Q_{c}$, the absolute minimum of $V_{\ell}$ lies at the larger $r^{2}$. The ground state wove function is then concentrated ot this rodius, which is very large if $\mathrm{g}$ is small. But the $0^{\text {th }}$-order of perturbation theory, which consists of taking $g=0$, gives a ground state concentrated at the much smaller $r^{2}$ (minimum of dotted eurve) and is therefore a very poor approximation. On the other hand, a very good approximation is obtained by using classical theory with the exact 9. This gives a circulas trajectory at the right radius, on which quanfum fluctuetions may be superimposed as corrections.

We note that os $g \rightarrow 0+$ at fixed $\Delta, q_{c}-\Delta^{2}$ and so if we moke $\Delta^{2}<1$ the situation described above occurs even for small quantum numbers.

We postulate that a similar situation orises from the interoction befween quarks and gluons; thot even for low quork numbers, the quantum bound stote is betrer oppreximated by a steady solution of the classical field equations than by the free-porticle state of the quantum theory without interactions. The stable bound state of the clossical theory is what we call a soliton.

Our solitons are stable only in isolation; they are what Iraditionol usage hos called "solitary waves". The traditional meaning of "soliton" is that of a canfiguration stable even against collisions; we propose to call such configurations "indestructible solifans", but do not onticipate any role for them in particle physics. We prefer to use the simpler ferm for the more opplicoble concept.

Our investigations are confined to nontopological solitons. This does not rule 
out o theory with degenerate vacua; it does mean that in such o theory we assume a single choice of vocuum at large distances, independent of direction.

Before these ideas are applied to a model of hodrons, f two questions should be . investigated. First, one wishes to know how widespread is the occurrence of solitons (i.e., stable, localized solutions of field equations) in classical field theories, and what the characteristic feotures are. Second, one may ask whether the classical soliton is indeed o good approximation to the exact quantum ground state in those few limiting coses where the latter is known.

The first principle in looking for nontopological solitons in a classicol theory of commuting fields is that stability connot be achieved with completely time-independent solutions. This is becouse the energy could alwoys be decreosed by shrinking the volume of the soliton while maintaining the peak field amplitude. To achieve steble solitons, we introduce o conserved "charge" associoted with a particle whose free moss we denote by $\mathrm{m}$.

If the "charged" porticle is a boson, we moy call the corresponding field $\Phi$. We pick a Lagrangian that depends on $\Phi$ only through $\Phi^{\dagger} \Phi$, and look for solutions in which $\Phi=e^{-i \omega t} \phi$, where $\phi$ and all other fields are time-independent. (More generally, we hove $\partial \Phi / \partial t=\omega(y \Phi)$ where $\dot{\theta}$ is a generator of some group an $\Phi$ that leaves the Lagrangian unchanged.) The "charge" $Q$ is then defined by $Q=\frac{\partial L}{\partial w}$. $Q$ is automatically conserved, and in the quantized theory it must be an integer. It plays the same role here as the angular momentum $\ell$ in the first-quantized illustration given previously. The energy $E$ moy now be minimized for fixed $Q$, since shrinking the volume would necessitole intensifying the field $\Phi$. After minimization we hove $\frac{d E}{d Q}=w$ when $Q$ is raken as a continuous variable. 
If the "chorged" particle is o fermion, we denole its field by $\psi$ and simply let $N=\int \psi^{t} \psi$ be the conserved fermion number. $N$ then plays the part of $Q$, and $\epsilon$ (the single-fermion energy in the presence of the soliton fields) plays that of w. After arriving at the field equations we can formally allow $N$ to vary continuously; then we hove $\frac{d E}{d N}=\mathrm{e}$.

The principal information we seek is the groph of Evs. $Q$ (or N). This curve is to be compored with the straight line $E=Q m$, which represents the energy of an aggregate of free particles bearing the some total charge. For stable soilitons to exist, the curve must lie below the straight line.

We recall that the slope of this curve is $w$, and it is easily seen from the field equations at lorge distances thof $w \leqslant m$. The behovior of $E$ vs $Q$ when $\xi \equiv \sqrt{m^{2}-\psi^{2}} \ll 1$ depends only on the dimensionality of spoce and not on the particular model. It is generally true in this limit that $\sim \xi$ and the radius $\sim \xi^{-1}$; hence $Q \sim \xi^{2} \xi^{-d}$ in $d$ spoce dimensions. The resulting curves for 1,2 , and 3 space dimensions ore shown in fig. 2; the low - w part of the eurve is amitted, In 1 or 2 dimensions, the curve con only lie under the straight line. In 3 dimensions the curve lies under the line when $Q$ exceeds some volue $Q_{S}$. In more detailed investigations we have found that the parcmeters can be chosen to make $Q_{S}<1$ if desired.

As w decreases to its lower limit win large. Hore we encounter two kinds of behovior, depending on the specific model. In some cases $\omega_{\min }>0$; the solution is then saturated in the sense that the amplitude of the charged field is roughly constont throughout the interior and does not 
increase as the rodius is made lorge. The energy then grows as $E \uparrow Q w$ min : (See Fig. 3a,) In other cases $\omega_{\text {min }}=0$. This leads to an unscrturated solution in which the chorged field grows without limit os the radius increoses. Generally we then hove $E \sim Q^{k}$, where $0<k<1$. (See fig. 3b.)

In 1 dimension solitons con arise from a single field. We put

$$
\begin{aligned}
& \mathcal{L}=\dot{\Phi}^{\dagger} \dot{\phi}-\phi^{\prime} t \Phi^{\prime}-U\left(\Phi^{\dagger} \phi\right)+\text { countertems, } \\
& m^{2}=\left.\right|_{\phi=0} \frac{d U\left(\phi^{2}\right)}{d \phi^{2}}, \\
& \phi=e^{-i \omega^{t}} \phi(x), \phi^{\prime 2}=U-\omega^{2} \phi^{2}, \\
& Q=\frac{\partial}{\partial \omega} f \mathscr{L} \mathrm{dx}=2 \omega \int \phi^{2} \mathrm{dx}, \quad \mathrm{E}=\int\left(\phi^{\prime}+U+\omega^{2} \phi^{2}\right) d x .
\end{aligned}
$$

At the turning point $\phi^{\prime}=0$, we must hove $U=\omega^{2} \phi^{2}$. Therefore the curve $U=U\left(\phi^{2}\right)$ must infersect the straight line $U=\omega^{2} \phi^{2} \cdot$. Since $\omega^{2}<m^{2}=U^{\prime}(0)$, the aurve $U\left(\phi^{2}\right)$ must hove a portion concove down. Several soluble examples are known:

$$
\text { (A) } U=m^{2} \phi^{2}\left[1-2 g^{2} \phi^{2}+g^{4}\left(1+\epsilon^{2}\right) \phi^{4}\right] \text {. }
$$

Here the curve cannot intersect the straight line unless $w>w_{\min }$ where $w_{\min }^{2}$ is the slope of the tongent shown in Fig. (4ai). Hence the behavior is soluroted at lange $Q$ (Fig. 4aii).

$$
\text { (B) } U=\frac{1}{4} \frac{m^{2}}{s^{2}}\left(\sqrt{1+B g^{2} \phi^{2}}-1\right) \text {. }
$$

Here $\omega$ can be as close to 0 os desired (Fig. 4bi). The behovior at large $Q$ is unsaturaled with $E \sim Q^{3 / 4}$ (Fig. 4bii). 


$$
\text { (C) } U=m^{2} \phi^{2} e^{-2 g^{2} \phi^{2}} \text {. }
$$

Another unsaturated cose, but here U decreases for large $\phi$ (Fig. 4ci) and hence the energy is concentrated at the boundary of the solitan. When $w-0$, the rodius grows longe but both $Q$ and $E$ remoin finite (Fig. 4eii) - an exception possible only in 1 dimension.

In 3 dimensions we restrict ourselves to 4 th-order polynomials for the sake of renormalization. This rules out pasitive definite functions $U\left(\phi_{t}^{2}\right)$ with a downward concavity as in the previous examples. But solitons are still possible with two fields.

We have studied two unsoturated examples:

$$
\begin{aligned}
& \text { (D) } \Phi \rightarrow \mathrm{e}^{-i \omega t} \Phi, \text { "charged" scolar boson, } \\
& X \text {, neutral scolar boson, } \\
& \mathcal{X}=-a_{\mu} \Phi^{\dagger} \partial_{\mu} \Phi-\frac{1}{2}\left(\partial_{\mu} X\right)^{2}-P^{2} X^{2} \Phi^{\dagger} \Phi-U(X)+\text { counterterms, }
\end{aligned}
$$

where $U$ has a minimum at $X=X_{\text {vac }}=\mathrm{m} / \mathrm{f}$. We then hove

$$
\begin{aligned}
& Q=2 \omega f \phi^{2} d^{3} r \\
& E=f\left[(\nabla \phi)^{2}+\frac{1}{2}(\nabla X)^{2}+f^{2} x^{2} \phi^{2}+U(X)+\omega^{2} \phi^{2}\right] d^{3} r
\end{aligned}
$$

ond by minimizing $E$ of fixed $Q$ we abtain coupled nenlinear 2nd-order differential equations in $\$$ and $X$. The E-Q curve (Fig. 5) has the spike characteristic of 3 dimensions. The stability threshold $Q_{S}$ can be mode $<1$ by suitably choosing the ratio of free masses. For lange $Q$ we have $E \sim Q^{3 / 4}$ unless $U$ has a specia! form making the vacuum degenerate with o second minimum of $X=0$. Then the energy is 
surfoce-dominated and $E \sim Q^{2 / 3}$ ot large $Q$.

$$
\begin{aligned}
& \text { (E) } \Psi \text {, "charged" spinar fermion } \\
& \sigma \text {, neutral scalar boson. }
\end{aligned}
$$

Here we adopt a slightly different formal opproach. We start from the Homiltorian density

where

$$
H z=H_{\sigma}+\sum_{k=1}^{n} \psi^{k \dagger} H_{D} \psi^{k}+\text { counterterms }
$$

$$
\begin{aligned}
& \boldsymbol{H}_{\sigma}=\frac{1}{2} \pi^{2}+\frac{1}{2}(\nabla \sigma)^{2}+U(\sigma), \pi \text { conjugate to } \sigma, \\
& H_{D}=\frac{1}{i} \vec{a} \cdot \vec{\nabla}+\beta(m+g \sigma)
\end{aligned}
$$

and $k$ is a "color" index which permits us to put up to $n$ quarks in the same orbital. We then opproximate the whale Hamiltonian by the "quasiclassical" Hamiltonian

$$
H_{q c 1}=f\left[\frac{1}{2}\left(\nabla \sigma_{c}\right)^{2}+U\left(\sigma_{c}\right)\right] d^{3} r+\sum_{k}^{n} \sum_{\ell}^{\infty} c_{\ell}\left(a_{l}^{k \dagger} o_{\ell}^{k}+b_{\ell}^{k \dagger} b_{\ell}^{k}\right)
$$

where the index $Q$ ronges over the eigenfunctions of $H_{D}$ with $\sigma=\sigma_{c}$, and $\epsilon_{Q}$. $o_{\ell}, b_{\ell}$ ore the corsesponding eigenvalues and annihilation operators for fermion and ontifermion. Here $\sigma_{c}$ is a c-number function, but the fermion field is still quanrized (the $a$ and b eperators obey anticommutolion rules).

The fermion number $N=\sum_{k} \sum_{\ell}\left(a_{\ell}^{k \dagger} a_{\ell}^{k}-b_{\ell}^{k \dagger} b_{\ell}\right)$ is conserved. We give it some fixed value $\leqslant n$; then the lowest eigenvalue of $H_{q c l}$ for a given $\sigma_{c}$ is

$$
E=N \epsilon+f\left[\frac{1}{2}\left(\nabla \sigma_{c}\right)^{2}+U\left(\sigma_{c}\right)\right] d^{3} r
$$


where $\epsilon$ is the lowest positive solution of

$$
\epsilon \phi_{c}=\left[\frac{1}{i} \vec{a} \cdot \vec{\nabla}+\beta\left(m+g \sigma_{c}\right)\right] \phi_{c} .
$$

We minimize $E$ with respect to $o_{c}$ and obtain

$$
\nabla^{2} \sigma_{c}-U\left(\sigma_{c}\right)=g N \psi_{c}^{\dagger} \beta_{c}^{\psi} .
$$

The qualitative properties of this soliton ore very similar to those of the provious example using only boson fields. In Fig. 5 , simply replace $Q$ by $N$.

We have also studied solurated examples in 3 dimensions.

(F) Some os (E) but with $N \gg n$.

This case has been studied by Lee and Wick ${ }^{(5)}$ and by Lee and Margulies ${ }^{(6)}$ as a model of nuclear matter. Here the nucleon density remains constant as $\mathrm{N}$ grows large, because of the Pauli principle. Both $N$ and $E$, in that limit, are simply proportional to volume. (See Fig. 6.)

(G) $\vec{V}_{\mu}$, Yang-Mills vector field

$\phi$, Higgs scalar, isospinor. (7)

The lograngion has $\mathrm{SU}_{2} \otimes \mathrm{SU}_{2}$ symmetry, with only the first group gauged. After the Higgs symmetry breaking, there remains one globel $\mathrm{SU}_{2}$ group under which is scolar and $\vec{V}_{\mu}$ is vector. Writing $\vec{V}_{\mu}=\left(\vec{V}_{k}, V_{0}\right)$, the time-dependence of the soliton is given by 


$$
\frac{\partial}{\partial t} \vec{V}_{k}=\vec{\omega} \times \vec{V}_{k}, \frac{\partial}{\partial t} \vec{V}_{0}=0, \frac{\partial}{\partial t} \phi=0 \text {. }
$$

The "charged" field is thus $\vec{V}_{k}$, and the scalor "glue" is provided by $\phi$. The 4th component $\vec{V}_{0}$ injects a new element: it mediotes a self-repulsion of $\vec{V}_{k}$ which leads to saturation as in Fig. 6 .

I now tum to the second question: how good is the quasiclossical approximation?

Let us define the binding energy $E_{B}=N E(1)-E(N)$ and the rotio $R=E_{B}$ (axact) $/ E_{B}(q e l)$ where $E_{8}(q e 1)$ is the value given by our soliton opproximations.

For relativistic theories, exact results are known only when $g^{2}<1.1$. When $N \gg 1$, in ail three cases (D), (E), and (F), we find $R=1$. For $N=2$, the exact result con be found by solving the Coulomb problem in cenfer-of-moss coordinates, and $\mathrm{R}=.768$ (3)

For nonrelativistic "chorged" particles we find also $R=1$ in the limit. $g^{2} \gg 1$ for any $N,(3)$ as well os for $N \gg 1$ and ony $g^{2}$. Again, when $N=2$ and $g^{2} \ll 1$, we have $R=.768$.

Either lorge $\mathrm{g}^{2}$ or large $\mathrm{N}$ terds to make the quantum corrections negligible, and even in the expected "worst" case $N=2, g^{2} \ll 1$, the approximation is not so bad. Thus we are encouroged to think that in a model of hodrons $(\mathrm{N}=2$ or 3 , $g^{2} \gg$ I but with relativistic binding) a quasiclassical treatment will be fairly occurote. The hadron model we have in mind involves an $\mathrm{SU}_{3} \otimes \mathrm{SU}_{3}$ holf-gouged symmetry leading to global $5 U_{3}$ after Higgs breaking, and four fields as shown in Table 1 : 
3

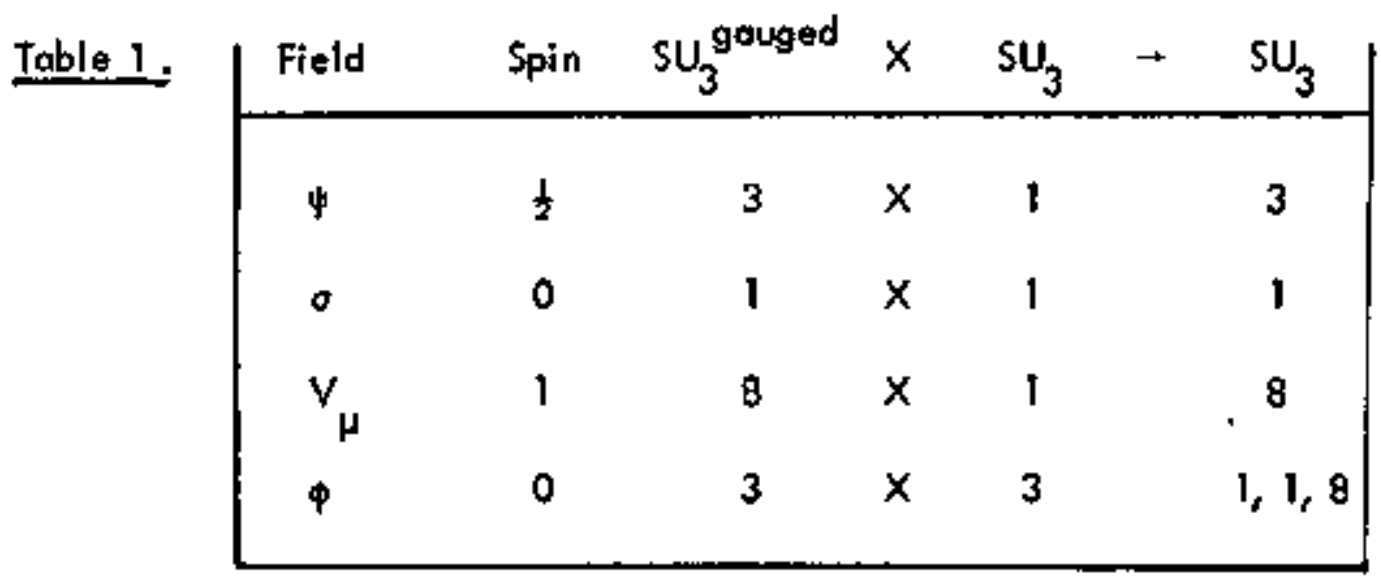

The $\$$ field represents the quark; its 3-fold symmetry is color. We have not yet injocted flavor. The o field is the scalar "glue". The vector field $V_{\mu}$, as suggested by Nambu, mediates a repulsive interaction raising the energy of color nonsinglets; thot explains why they are not seen. The Higgs field $\phi$ is then needed to give the $\vec{V}_{\mu}$ a mass in the usual way. All the free masses are ossumed large, so that the observed spectrum is merely that of the color-singlet bound stotes.

In the soliton approximotion, o color-singlet stote hos $\vec{V}_{\mu}=0$ everywhere. The $\phi$ field does not interact directly with $\psi\left(\phi^{2} \phi_{\psi}^{\dagger}\right.$ is not renomalizable, while $\phi \psi^{\dagger} \psi$ breaks the pre-Higgs symmetry) and its effect on a can presumebly be incorporated into $U(\sigma)$. Thus the bound-state problem simplifies to that of excmple (E) above, with just $\Psi$ and $\sigma$, and $N=2$ or 3 . It is the same model that led to the SLAC bog, ${ }^{(8)}$ and with different porometers was used by Creukz ond Soh ${ }^{(9)}$ to give the MIT bog $(10)$ in a cerfain limit.

There are four main styles of bag, choracterized by (a) either MIT- or SLACtype quark wave function (see Fig. 7) and (b) either valume- or surface-dominated o energy. See Table 2. 


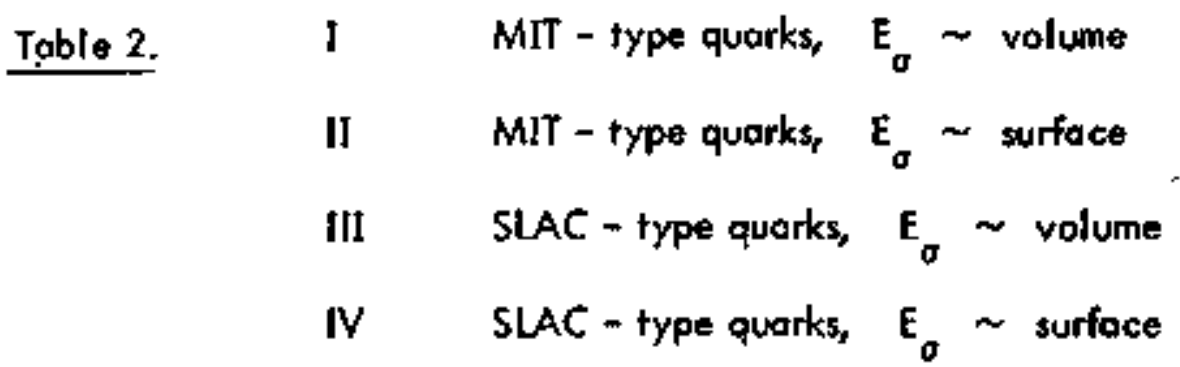

To obtain I or If from the field model, one sets (with Creutz and 5oh) $m+g \sigma_{0}=0$ where U(o) has its second minimum of $\sigma=\sigma_{0}<0$. To obtain III or IV, one sets $m+g \sigma_{0}<0$. For I or III, one must have $U\left(\sigma_{0}\right)>U(0)$, for II or N $U\left(\sigma_{0}\right)=U(0)$. The published MIT bog is I, the SLAC bog is IV.

If $m+g \sigma=0$, before passing to the MIT limit, one can olso obtain hybrid quark wave functions as in Fig. 8. These arise from a tendency for $\sigma$ to overshoot the value $v_{0}$ so as to give the quark the lower SLAC-type energy. This effect con be seen in the curves of Rofelski for the case $\mathrm{U} \sim \sigma^{2}$. If an $\mathrm{SU}_{3}$ flavor group with unbroken symmetry is introduced, the model gives 36 degenerote mesons, and 56 baryons.

Some preliminary numerical comparisons ore shown in Table 3. (Some of these numbers are already known.) Letting

$$
\begin{aligned}
m & =\text { free quark mass } \\
\mu & =\text { free gluon mass } \\
m_{B} & =\text { average (56) baryon moss } \\
& =1.40 \mathrm{~m} \mathrm{~N} \\
B_{-} & =U\left(\sigma_{0}\right) \rightarrow B \text { of MIT bag } \\
B_{+} & =\max U(\sigma) \text { for } 0>\sigma>\sigma_{0}
\end{aligned}
$$




$$
\xi=\left(\frac{m_{B}}{m}\right)^{2}, \quad \eta=\frac{m_{B}}{\mu}, \quad \zeta=\frac{B_{-}}{B_{+}},
$$

we ossume $\xi, \eta, \zeta$ all $\ll 1$ and obtain cose

I when $\xi \gg \eta \gg \zeta$
II when $\xi \gg \zeta \gg \eta$
IIt when $\zeta \gg(\xi, \eta)$
IV when $\eta \gg(\xi, \zeta)$.

\begin{tabular}{|c|c|c|c|c|c|}
\hline Quantiły & Expt. & 1 & It & III & IV \\
\hline$m_{N}\left\langle r^{2}\right\rangle^{\frac{1}{2}}$ & 3.86 & 4.25 & 4.78 & 2.86 & 3.21 \\
\hline $2 m N \mu_{p}$ & 2.79 & 2.36 & 2.66 & 1.90 & 2.14 \\
\hline$\mu_{n} / \mu_{p}$ & -.685 & $\longleftarrow$ & & $-2 / 3$ & $\longrightarrow$ \\
\hline$g_{\Lambda} / g_{V}$ & 1.2 & $\leftarrow 1$. & $\longrightarrow$ & $\leftarrow$ & $\rightarrow$ \\
\hline
\end{tabular}

Toble 3

The main result of the comporison is that all the numbers are in the general vicinity of experiment, although the MIT-type models seem somewhot closer. We mean to investigate the hybrid models as well.

Further questions: How do we colculate form foctors from such a model? How should we intraduce flavor breoking? Does 'PCAC come in at all?

To conclude: This opproach seems the most conservative of all ovenues to the study of hadron structure. At the same time it incorporates most of the features definitely indicated by the research of the last fifteen years. Since a preliminory comporison with 
13.

experiment leads to no glaring controdiction, the opproach should be pursued untit

it leads either to a significant success or to an informative foilure. 
3

\section{References}

1. T. D. Lee, of the Symposium on Frontier Problems in High Energy Physics, Piso, 1976 [Columbia University preprint No, CO-2271-76].

2. R. Friedberg, T. D. Lee and A. Sirlin, Phys, Rev. D13, 2739 (1976).

3. R Friedberg and T. D. Lee, Phys, Rev, D15, 1694 (1977).

4. A. Nishimura, University of Tokyo preprint UT-275.

S. T. D. Lee and G. C. Wick, Phys, Rev. D9, 2291 (1974).

6. T. D. Lee and M. Margulies, Phys, Rev. D11, 1591 (1975).

7. R. Friedberg, T. D. Lee and A. Sirlin, Nucl. Phys, B115, 1, 32 (1976).

8. W. A, Bardeen, M. S. Chanowitz, S. D. Drell, M. Weinstein and T. M Yan, Phys. Rev. D11, 1094 (1975).

9. M. Creutz and K. S. Soh, Phys. Rev. D12, 443 (1975).

10. A. Chodos, R. L. Joffe, K. Johnson, C. B. Thom and V. F. Weisskopf, Fhys.Rev. D9, 3471 (1974). 


\section{FIGURE CAPTIONS}

Figure $1 \quad V_{x}=V+\frac{\ell^{2}}{2 \tau^{2}}$ against $r^{2}$ in simple 1st quantized exomple

Figure $2 \quad E$ vs $Q$ in upper ronge of $\omega$

Figure $3 \quad E$ vs $Q$ in lower range of $\omega$

Figure 4 Solitons in 1 dimension

Figure 5 Unsaturoted 3-dimensionol examples

Figure 6 Satturated 3-dimensional examples

Figure $7 \quad$ (o) MIT-like Dirac wove function

(b) SLAC-like Dirac wave function

$2 \quad$ Figure $8^{-} \quad$ Hybrid Dirat wave funtion 


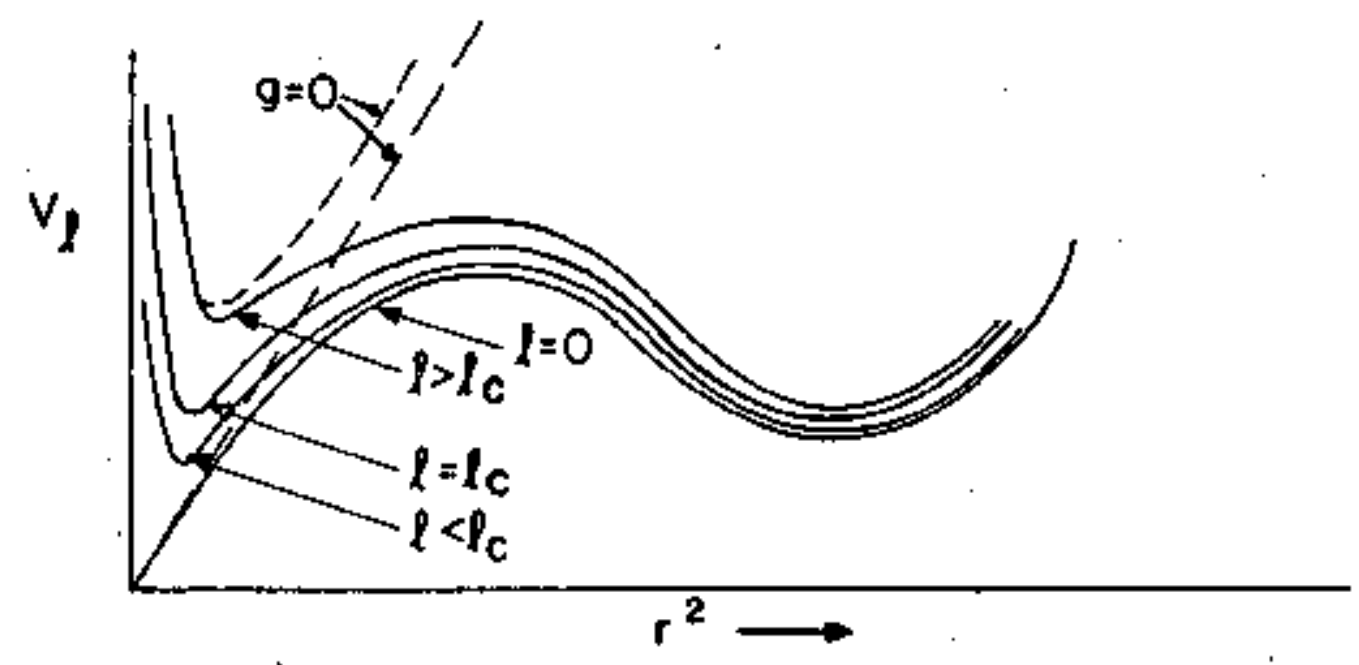

FIG. I

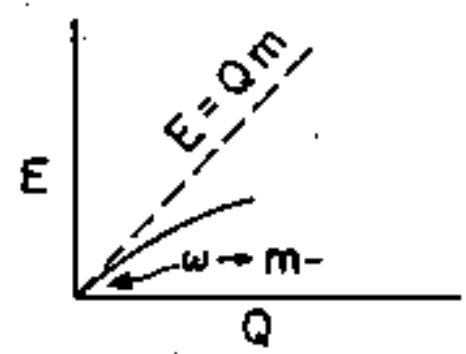

1 dim.

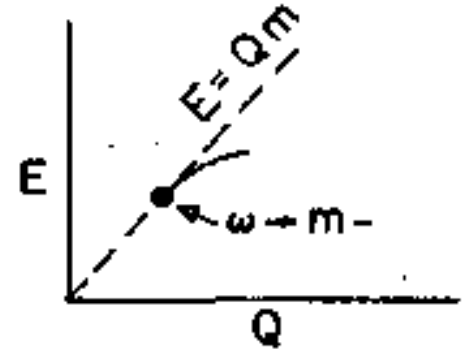

2 dim.

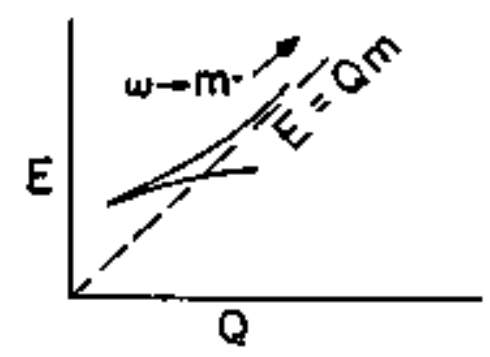

$3 \mathrm{dim}$.

FIG: 2

(a) SATURATED

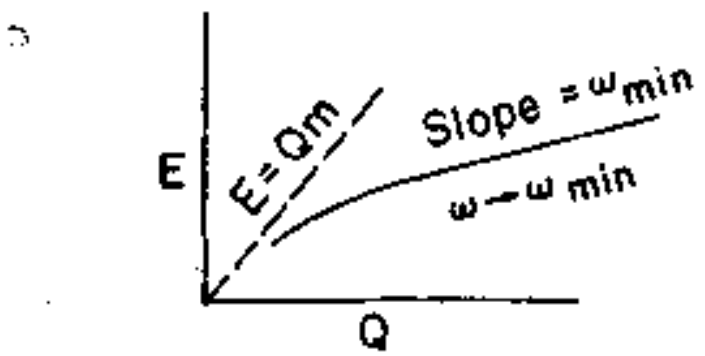

(b) UNSATURATEO

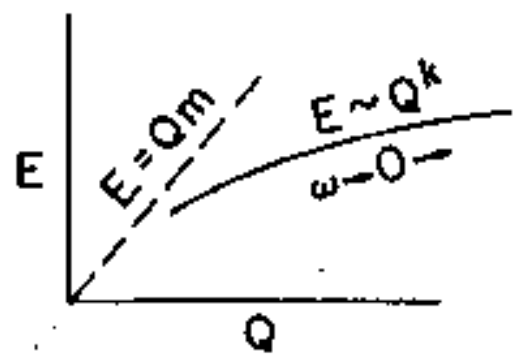

FIG. 3 


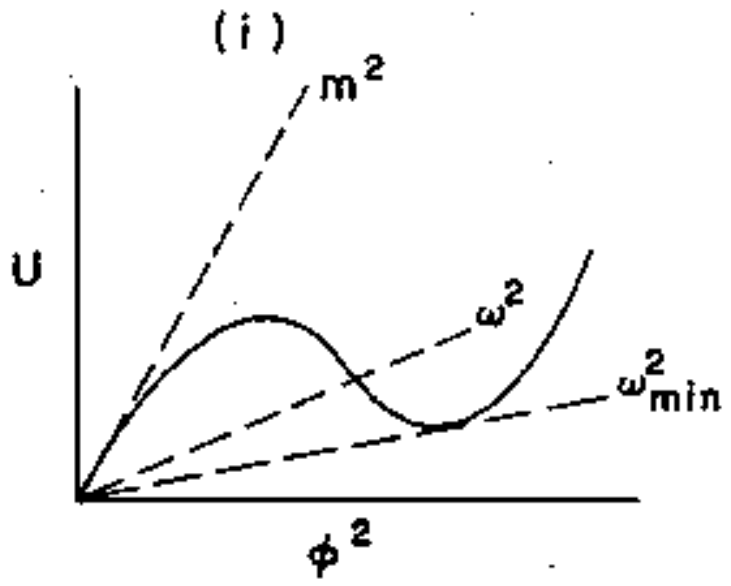

(a)
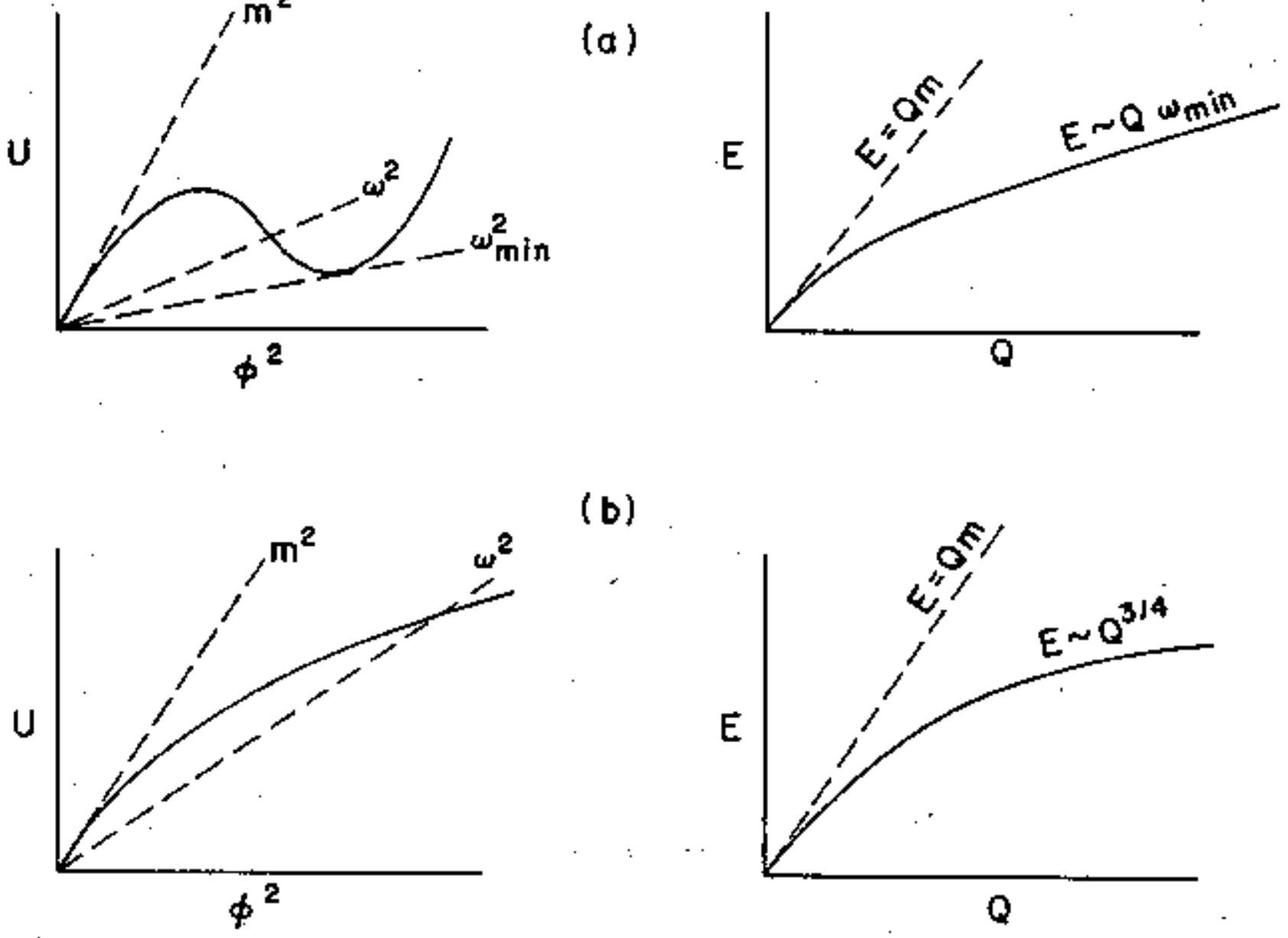

(b)

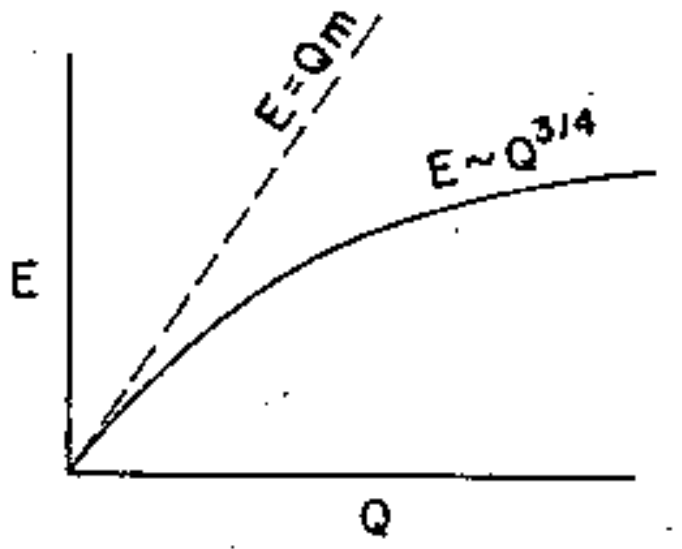

3

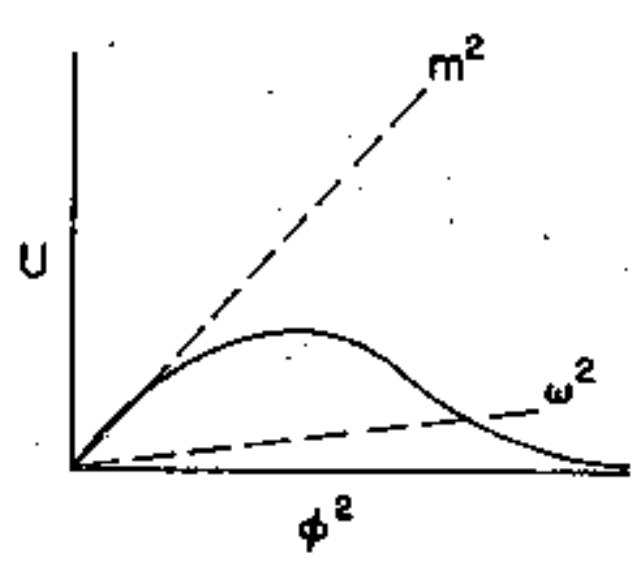

(c)

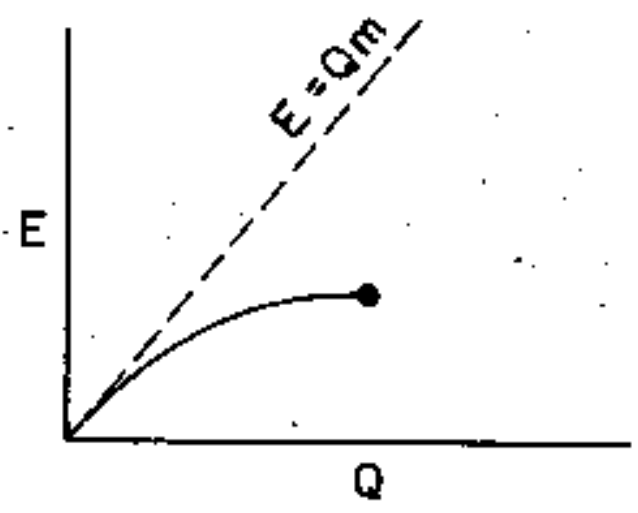

FIG. 4 

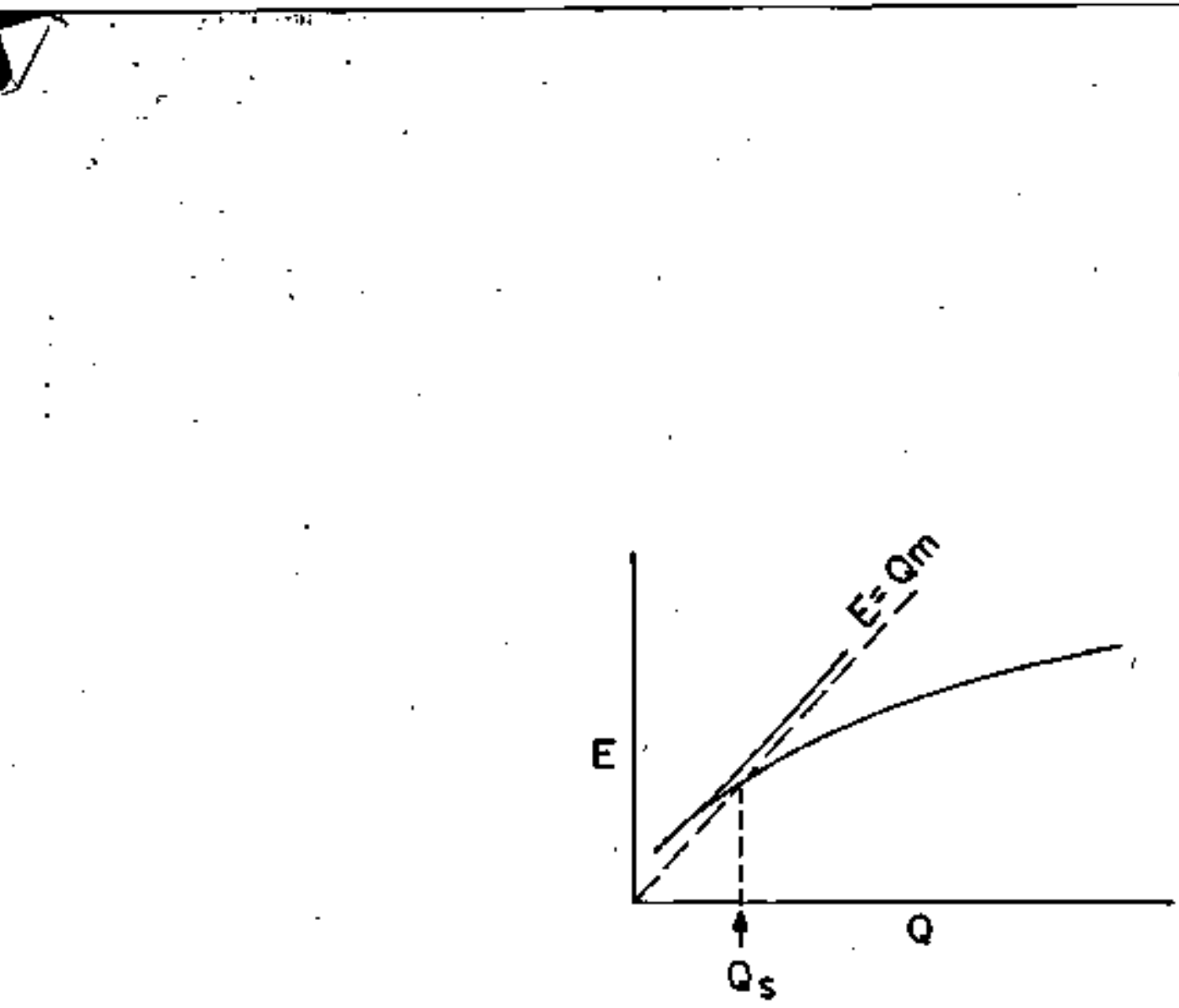

FIG. 5

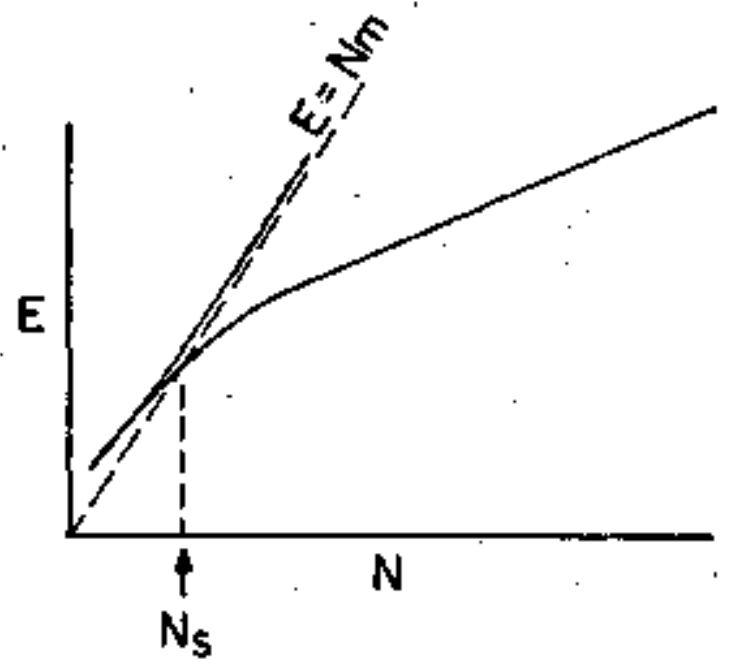

FIG. 

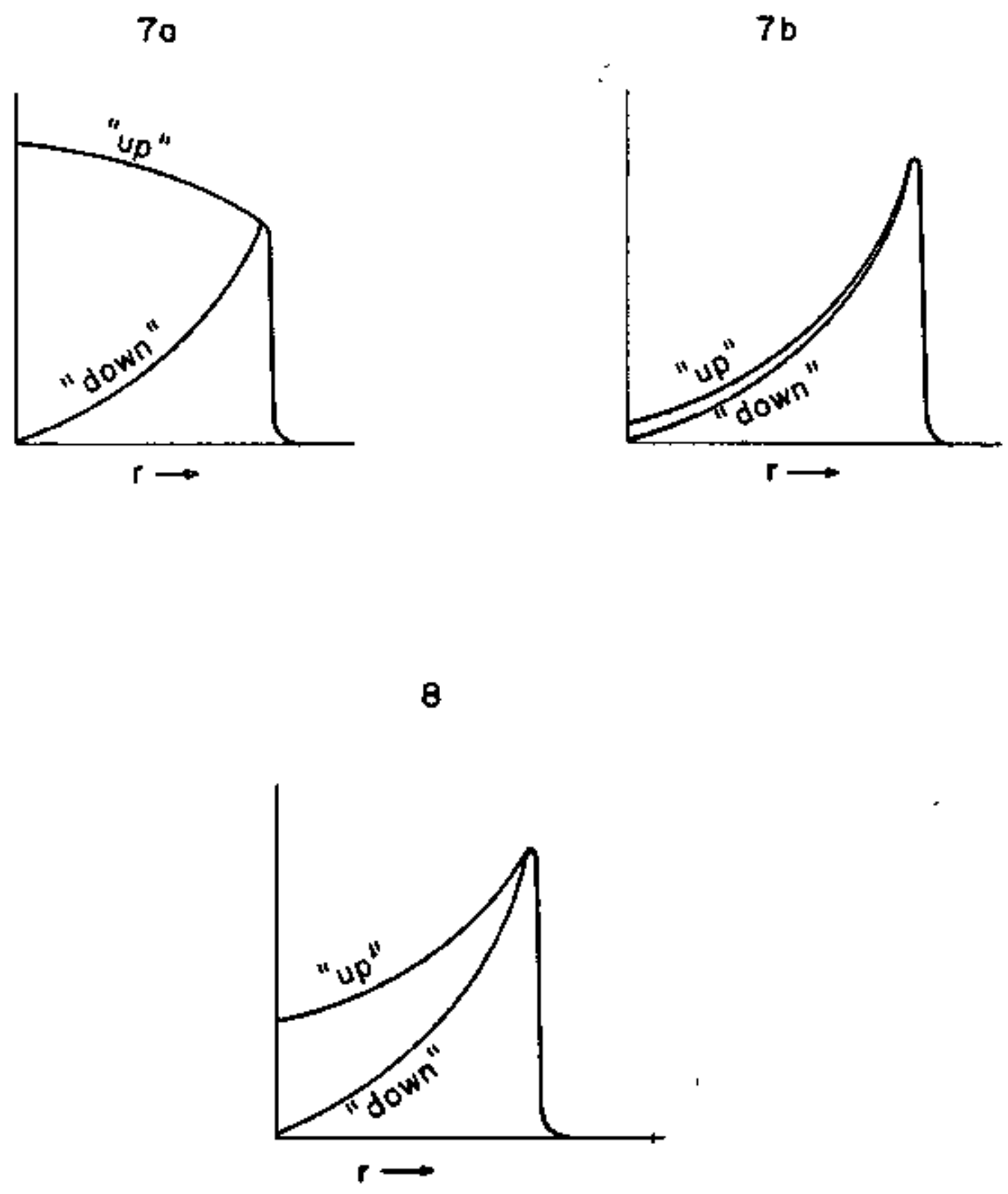